\title{
Rapamycin and dexamethasone during pregnancy prevent tuberous sclerosis complex-associated cystic kidney disease
}

\author{
Morris Nechama, ${ }^{1}$ Yaniv Makayes, ${ }^{1}$ Elad Resnick, ${ }^{1}$ Karen Meir, ${ }^{2}$ and Oded Volovelsky ${ }^{1}$ \\ ${ }^{1}$ Pediatric Nephrology Unit and ${ }^{2}$ Department of Pathology, Hadassah-Hebrew University Medical Center, Jerusalem, Israel.
}

Chronic kidney disease is the main cause of mortality in patients with tuberous sclerosis complex (TSC) disease. The mechanisms underlying TSC cystic kidney disease remain unclear, with no available interventions to prevent cyst formation. Using targeted deletion of TSC1 in nephron progenitor cells, we showed that cysts in TSC1-null embryonic kidneys originate from injured proximal tubular cells with high mTOR complex 1 activity. Injection of rapamycin to pregnant mice inhibited the mTOR pathway and tubular cell proliferation in kidneys of TSC1-null offspring. Rapamycin also prevented renal cystogenesis and prolonged the life span of TSC newborns. Gene expression analysis of proximal tubule cells identified sets of genes and pathways that were modified secondary to TSC1 deletion and rescued by rapamycin administration during nephrogenesis. Inflammation with mononuclear infiltration was observed in the cystic areas of TSC1-null kidneys. Dexamethasone administration during pregnancy decreased cyst formation by not only inhibiting the inflammatory response, but also interfering with the mTORC1 pathway. These results reveal mechanisms of cystogenesis in TSC disease and suggest interventions before birth to ameliorate cystic disease in offspring.

Conflict of interest: The authors have declared that no conflict of interest exists.

Copyright: (ㄷ) 2020, American Society for Clinical Investigation.

Submitted: February 3, 2020

Accepted: May 27, 2020

Published: July 9, 2020.

Reference information: /CI Insight. 2020;5(13):e136857.

https://doi.org/10.1172/jici.

insight.136857.

\section{Introduction}

Tuberous sclerosis complex disease (TSC) is a genetic disorder affecting various organs, including the brain, kidney, skin, and heart, with an estimated prevalence of approximately 1:6,000 in all populations studied (1-4). De novo or inherited autosomal dominant mutations in TSC1 or TSC2 result in inactivation of TSC composed of hamartin and tuberin proteins, respectively. The complex acts as a tumor suppressor and inhibits the activity of mTOR complex 1 (mTORC1), a central regulator of various cellular functions such as protein translation, proliferation, metabolism, and autophagy (5-7). However, the hamartin-tuberin complex also has mTORC1-independent cellular effects through distinct pathways. For instance, we have previously shown that the effect of improved nephron endowment by TSC1 hemizygous deletion in nephron progenitor cells (NPCs) is independent of mTORC1 activity $(8,9)$. Interruption of the hamartin-tuberin complex by TSC2 deletion also has mTORC1-independent effects on prostaglandin production and NOTCH activity (10-12). PAK2 and TGF- $\beta-S m a d 2 / 3$ signaling pathways were suggested to mediate this independent effect as well $(13,14)$. Moreover, in cancer cells, TSC2 was shown to regulate VEGF gene expression in a mTOR-independent pathway (15). Indeed, some of the clinical manifestations of TSC disease respond only partially to mTOR inhibitors $(2,13,14,16,17)$.

TSC has debilitating neurological effects in childhood, including convulsions and autism spectrum disorder, which usually stabilize in late adolescence $(18,19)$. Chronic kidney disease (CKD) is the main cause of morbidity in adult patients with TSC $(20,21)$. The renal manifestations of TSC include renal angiomyolipoma (AML), benign vascular lesions, and cystic disease of the kidneys, which appear in the majority of patients $(21,22)$. AML of large dimensions poses an increased risk of bleeding if left untreated. Cystic kidney disease leads to a gradual loss of renal parenchyma, aggravated by the decline in nephron number consequent to multiple surgical procedures for AML resections and ablations. As a result, patients with TSC are exposed to complications of CKD at an earlier age than the general population. About $40 \%$ of patients with TSC eventually develop advanced CKD (stage III-V) (23). The burden of CKD in patients with TSC ranges in severity from a single renal cyst to a severe polycystic phenotype, especially in deletion disrupting both TSC2 and PKD1 genes and PKD1 $(24,25)$. The precise pathomechanism by which this autosomal 
dominant disorder causes cystic renal disease remains poorly understood. Furthermore, although mTOR inhibitors reduce the size and bleeding risk of large $\operatorname{AML}(16,26)$, their therapeutic effects and, even more critical, their preventive effects on TSC cystic kidney disease are still controversial $(21,27,28)$.

We have previously generated a mouse model of TSC by specific deletion of TSC1 in NPCs using CreLox recombination in mice (8). NPCs differentiate into the various segments of the nephron, forming the basic filtering unit. They originate from the embryonic metanephric mesenchyme and disappear in the third postpartum day in mice, with no subsequent regeneration. Although TSC1 hemizygous deletion induces nephrogenesis, complete deletion of TSC1 leads to a lethal cystic phenotype characterized by severe injury to the renal proximal tubular cells (PTCs) (8).

We now show that mononuclear inflammation, especially macrophage infiltration, plays a central role in cystogenesis in CKD of TSC. The cyst formation is paralleled by increased mTOR activity and cystogenic processes, such as increased c-Myc expression and cell proliferation. Furthermore, the cystic disease in offspring can be prevented using mTOR inhibitors and corticosteroids during pregnancy. Our findings identify mechanisms and therapeutic targets to overcome TSC cystic kidney disease.

\section{Results}

We have previously shown that complete deletion of TSC1 in NPCs induces tubular damage as early as E15.5 with swollen cellular appearance and occluded lumen. Cyst formation was evident at E17.5 (Figure 1, A and B) (8). Dissection of kidneys from TSC1-null embryos at various time points during embryogenesis herein identified increased phosphorylation of ribosomal protein S6 (pS6), the main downstream target of mTORC1 at E15.5 and E17.5 (Figure 1C). The increased levels of pS6 were evident in the damaged PTCs, as was determined by lotus tetragonolobus lectin-positive (LTL-positive) cyst-lining epithelial cells (Figure 1D and Supplemental Figure 1; supplemental material available online with this article; https:// doi.org/10.1172/jci.insight.136857DS1). The cysts appeared in the late stages of pregnancy, beginning at E17.5. Therefore, the increased pS6 staining initially appeared in the injured tubular cells and then in the cyst-lining epithelial cells, which originate from the PTCs. Therefore, TSC1 deletion in NPCs leads to proximal tubular injury and cyst formation, associated with mTORC1 activation.

Next, we examined whether mTORC1 inhibition alleviates TSC tubular damage and cystogenesis. $T S C 1^{f l f l}$ female mice were mated with Six2 $\mathrm{Cre}^{t g /+} \mathrm{TSC1}^{f / /+}$ males to generate Six2 $\mathrm{Cre}^{t g /+}$ TSC1 $^{f l / f l}$ pups with NPC-specific TSC deletion (25\% of offspring). We injected pregnant $T S C 1^{f / f l}$ females with rapamycin, a potent and specific inhibitor of mTORC1 or vehicle, on embryonic days E12.5, E14.5, and E16.5 (Figure 2A). We measured tubular damage and cyst formation in kidneys of the $\mathrm{TSC1}^{-/-}$offspring. Rapamycin prevented cellular injury and cystogenesis, as demonstrated by a decline in cyst area and number (Figure 2, B and C). While mTOR activity dramatically increased in $\mathrm{TSC1}^{-1-}$ animals compared with control littermates, rapamycin decreased mTORC1 activity, as shown by reduced pS6 levels in both null and hemizygous TSC1 kidneys compared with controls. pS6 levels decreased by both immunofluorescent (IF) staining and Western blot analysis (Figure 2, B, D, and E, and Supplemental Figure 3).

$\mathrm{c}-\mathrm{Myc}$ is a potent transcription factor involved in diverse cellular activities, previously demonstrated to be involved in cystogenesis in autosomal dominant polycystic kidney disease (ADPKD) $(29,30)$. In addition to the increase in mTORC1 activity, c-Myc levels increased in both hemizygous and TSC1-null kidneys. Furthermore, c-Myc predominantly expressed in cyst-lining epithelial cells (Figure 2B). Rapamycin decreased c-Myc levels, as observed by both IF staining and Western blot analysis (Figure 2, B, D, and E). Cell proliferation was also inhibited by rapamycin, as measured by decreased expression of the proliferation marker Ki67. Therefore, rapamycin ameliorates cystic kidney disease by reducing mTOR activity, c-Myc expression, and cell proliferation, all of which have been previously shown to be involved in cyst formation in other renal cystic diseases $(29,30)$.

NPC-specific TSC1-null mice die 2 days after birth (8). We therefore investigated whether rapamycin improves the survival of TSC mice. To that end, we monitored the effect of rapamycin administered during pregnancy on offspring survival. Rapamycin prolonged the survival of pups up to the age of P14, demonstrating a significant effect of rapamycin after birth (Figure 2F). At P14, the effect of rapamycin was no longer evident, and the mice had a high cystic burden and increased pS6 expression in the proximal tubules due to TSC1 ablation (Supplemental Figure 2). Thus, rapamycin administration during pregnancy prolonged the life span of newborn TSC mice by transient inhibition of renal tubular damage and cystogenesis. 

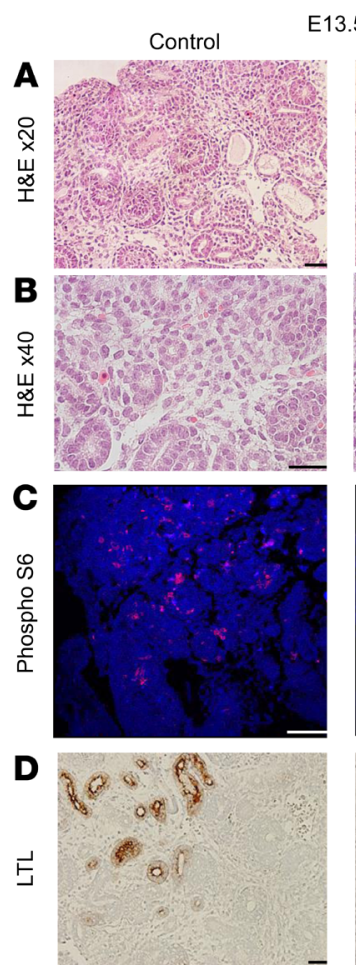

Six2 Cre TSC1ff
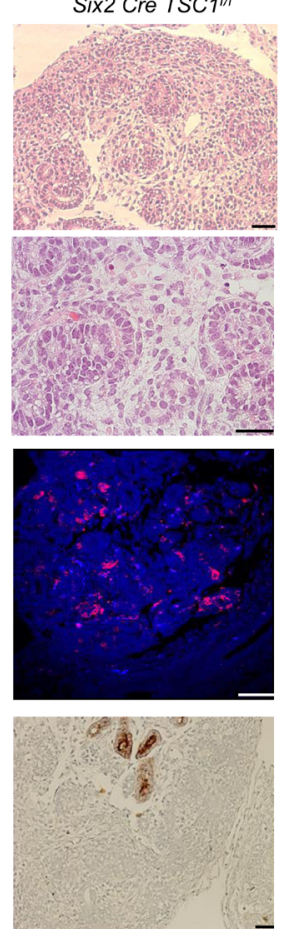

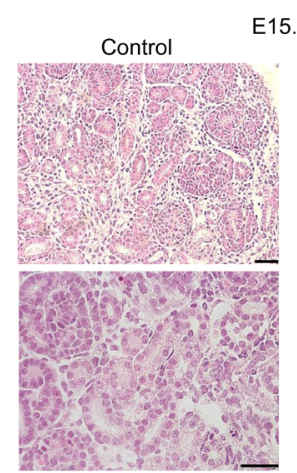

E15.5
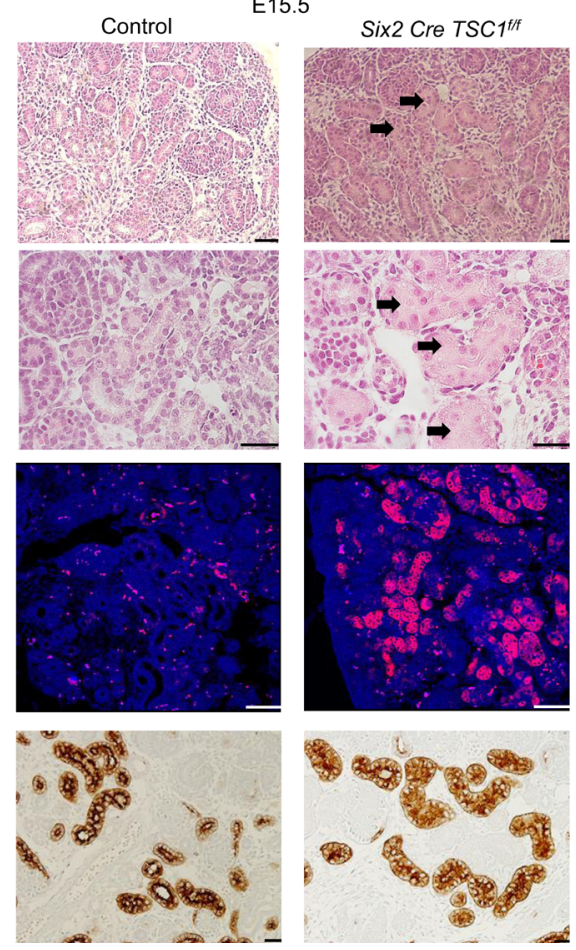

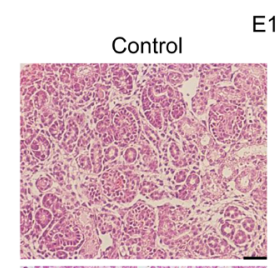

E17.5 Six2 Cre TSC1fif
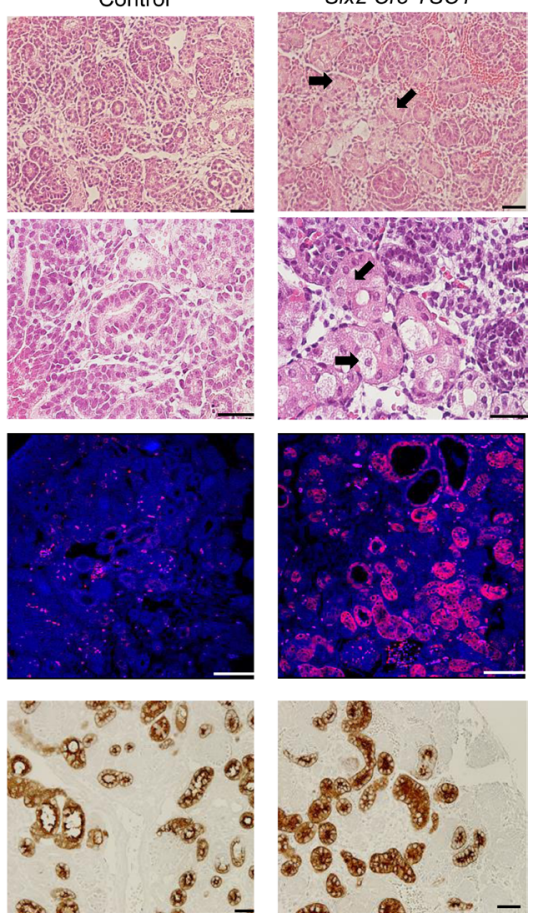

Figure 1. TSC1 deletion in NPCs is associated with mTOR pathway activation in PTCs. Kidney sections at different embryonic ages as indicated were stained with (A) H\&E. (B) Enlarged kidney sections as in A. (C) Phosphorylated S6 analyzed by IF. (D) LTL antibody by IHC. Scale bar: 50 4m. $n=3$. Black arrows indicate the injured PTCs with swollen appearance and occluded lumen. TSC, tuberous sclerosis complex; NPCs, nephron progenitor cells; PTCs, proximal tubular cells; IF, immunofluorescent; LTL lotus tetragonolobus lectin.

We then examined the effect of TSC1 deletion on global gene expression and intracellular pathways in PTCs and the effect of rapamycin on both. For this purpose, we sort Prominin1-positive PTCs from control and Six2 $C r e^{t g /+} T S C 1^{f / f l}$ mice (31), with and without rapamycin as above (Figure 2A and Figure $3 \mathrm{~A})$. Gene expression was analyzed by RNA sequencing to identify genes and pathways that are affected by TSC1 deletion and rescued by rapamycin treatment during pregnancy (Figure 3B). Among these pathways, we found an increase in the expression of genes related to inflammatory response and complement pathway activation in TSC1-null mouse PTCs compared with controls. The expression of these genes was downregulated in PTCs from pups of mothers injected with rapamycin during pregnancy (Figure 3, C-E). Many of these genes are associated with macrophage polarization and chemotaxis to the inflammation site (32-34).

Inflammation with high mononuclear cell infiltration was evident in cystic kidneys of TSC1-null P14 mice (Figure 2G). Cystic kidneys of TSC1-null P0 mice demonstrated increased levels of the NF-kB P65 subunit protein (Figure 4, A-C). Increased macrophage infiltration in P0 mice was also demonstrated by high levels of the F4/80 macrophage marker expression by immunostaining as well as high levels of F4/80 cells in dissociated TSC1 ${ }^{-1-}$ kidneys, as reflected by FACS analysis (Figure 4, A and D). Therefore, cystic kidneys in TSC show an inflammatory response and macrophage infiltration.

We next examined whether antiinflammatory treatment during pregnancy prevents the TSC cystic disease in a similar manner as mTOR inhibition. Corticosteroids are potent antiinflammatory medications that are frequently used during high-risk preterm pregnancies for various indications, including acceleration of lung maturation (35). Dexamethasone was injected to pregnant TSC mice carrying TSC1-null embryos, at the same time course as rapamycin (E12.5, E14.5, and E16.5) (Figure 2A). Dexamethasone administration during gestation improved renal cystic burden (Figure 4, E and F) and significantly reduced macrophage infiltration, as indicated by F4/80 immunostaining and FACS analysis (Figure 4, E, G, and $\mathrm{H})$. However, dexamethasone did not rescue the injured proximal tubular morphology, as these still demonstrated swollen epithelial cells and occluded lumens. Dexamethasone decreased mTORC1 activity but not P65 in TSC cystic kidneys (Figure 4, I-K). Therefore, inflammation plays a central role in the pathogenesis 
A

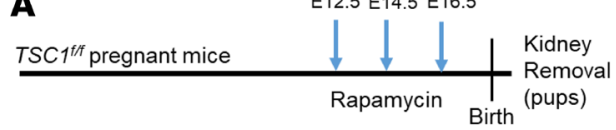

B
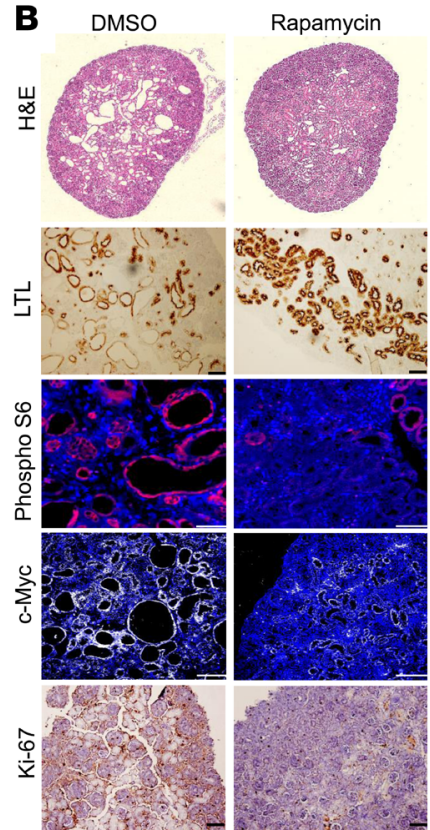

C

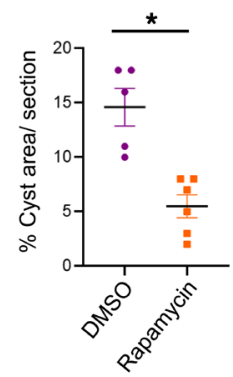

E

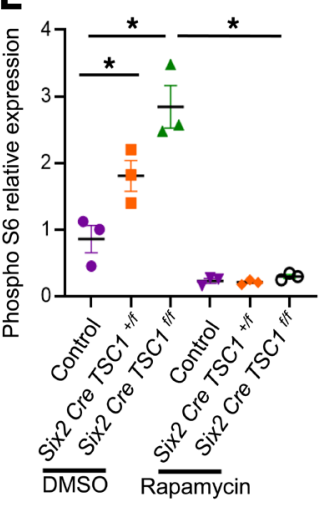

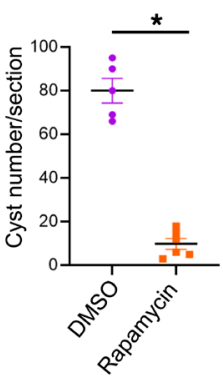

D
GAPDH
Phospho S6
C-Myc

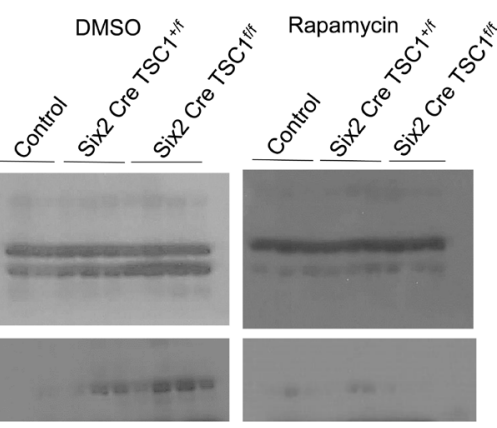

$\mathbf{F}$

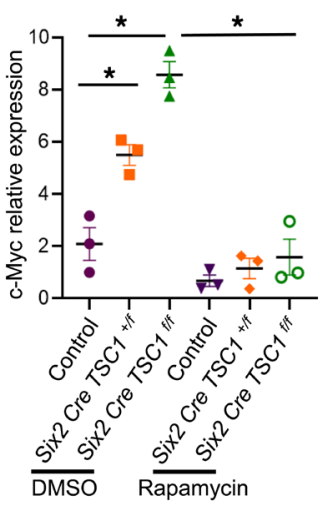

DMSO -

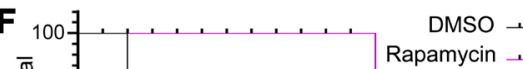

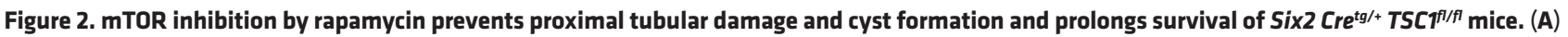
Experimental time course. $T S C 7^{f / f l}$ females were mated with Six2 $\mathrm{Cre}^{\mathrm{tg} /+} \mathrm{TSC}^{\mathrm{fl} /+}$ males. Rapamycin or vehicle (DMSO) was injected at the indicated gestational

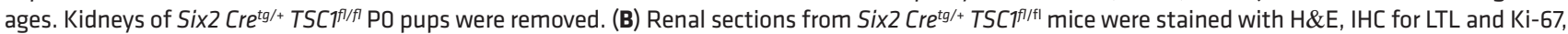
and IF for phospho-S6 (pS6) and c-Myc. Scale bar: $50 \mu \mathrm{m} . n=3$ in each group. (C) Quantitative analysis of cyst area and number of cyst per section as in B. ${ }^{*} P$

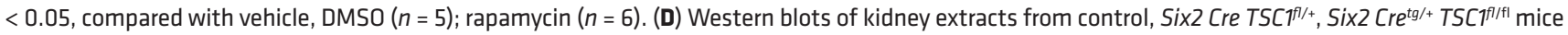
treated with rapamycin or vehicle as in A, analyzed for pS6, GAPDH, and c-Myc, showing elevated pS6 and c-Myc expression in heterozygous and homozygous renal extracts compared with control and reduction in the expression of these proteins upon rapamycin treatment. (E) Quantification of the Western blot as in D. ${ }^{*} P<0.05$, compared with control (WT). $n=3$ in each group. (F) Kaplan-Meier curve showing improved survival rate of rapamycin-treated Six2 Cre ${ }^{t g /+}$ TSC $7^{f / f l}$ mice $(n=6)$, compared with DMSO $(n=6)$. (C) H\&E staining of P14 offspring of rapamycin-treated Six2 Cre ${ }^{t g /+} T S C 7^{f / f l}$ mice as in A, demonstrating mononuclear infiltrate. The dashed circle indicates mononuclear inflammation site. Scale bar: $500 \mu \mathrm{m} . n=3$. Unpaired $t$ test was used for C and 1-way ANOVA statistical analysis was used followed by Duncan's post hoc test for $\mathbf{E}$. TSC, tuberous sclerosis complex; IF, immunofluorescent; LTL lotus tetragonolobus lectin.

of cystic TSC kidney disease and corticosteroid administration during gestation prevents cystogenesis in TSC by inhibiting both mTOR activation and inflammation.

\section{Discussion}

In our TSC mouse model, TSC1 deletion in NPCs induced proximal tubule cell damage and cyst formation, starting as early as E15.5. Based on our findings, mTORC1 hyperactivation in TSC-null proximal renal tubules leads to tubular cell damage and proliferation, also through c-Myc activation. Administration of steroids during pregnancy prevented cyst formation in TSC offspring by not only hindering the inflammatory process, as the high macrophage infiltration, but also downregulating mTORC1 activity (Figure 5).

Various murine models were based on TSC1 or TSC2 deletions in distinct portions of the nephron. In the current model, elimination of both TSC1 alleles was introduced specifically in the progenitor cells, and therefore it was present in most segments of the nephron in the offspring. Using this strategy we also showed that the resulting cysts originate from the proximal tubules. Moreover, the severe cystic disease appeared only after homozygous deletion of TSC1 in our model. It has been previously suggested that a second hit, which leads to loss of heterozygosity, takes place in the cystic cells as reported in TSC-AML and pulmonary lymphangiomatosis. However, the reports on loss of heterozygosity in TSC renal cysts are not consistent $(36,37)$.

Rapamycin has been successfully used during pregnancy in small clinical trials to prevent TSC manifestations, such as cardiac tumors $(38,39)$. Benefits from the early use of rapamycin were also demonstrated in neurological manifestations of TS, such as developmental delay (40). However, the safety of rapamycin during pregnancy has not been studied thoroughly in human studies and teratogenic effects have been 
A
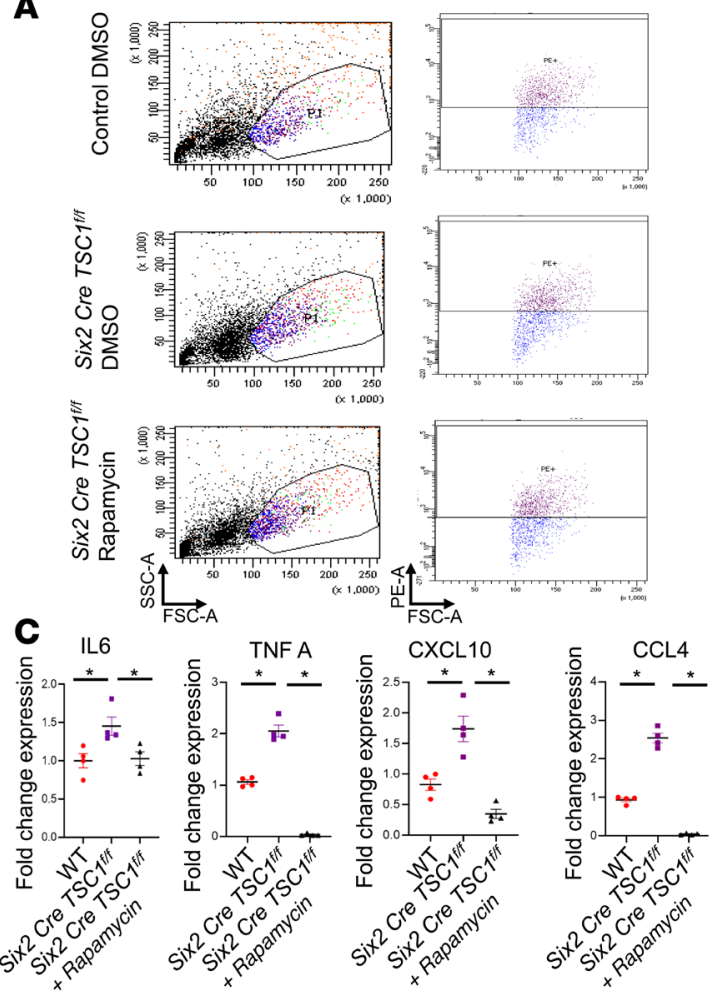

B

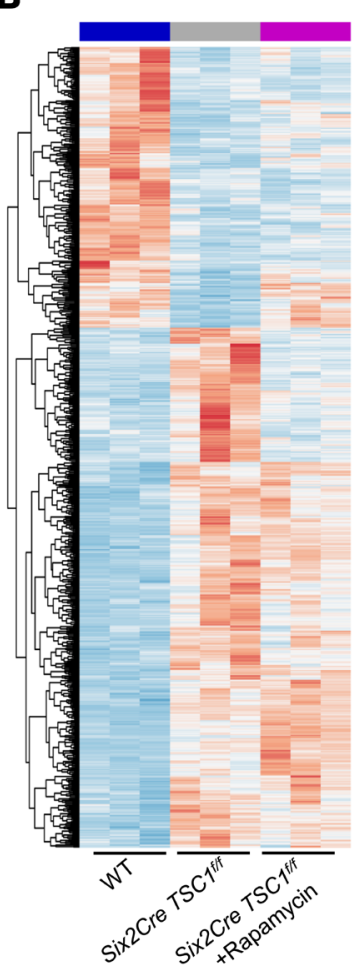

D

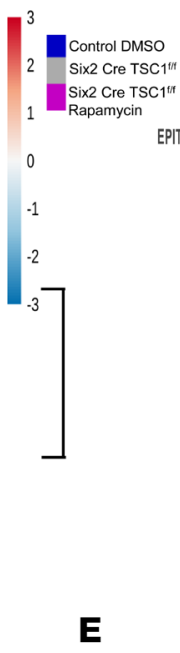
LL2 STATS SIGNALLNG
TNFA SIGNALING VIA NFKB HYPOXIAA
APOPTOSIS APOPTOSIS-
UVRESPONSE DNAPOPTOSIS-
UVRESPONSE DN-
TGF BETA SIGNALING
BHELIAL MESENCHYMAL TRANSTION-

$\mathbf{E}$
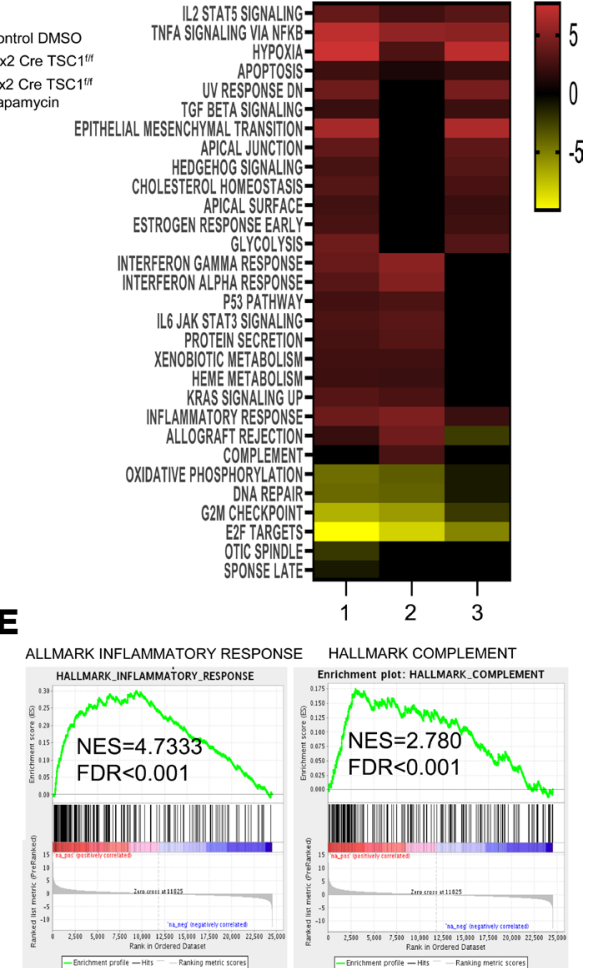

INTERFERON GAILYCOLYSIS INTERFERON GAMMA RESPONSENTERFERON ALPHA RESPONSEIL6 JAK STAT3 SIGNALLNG PROTEIN SECRETION HEME METABOLIS KRAS SIGNALING UP INFLAMMATORY RESPONSEALLOGRAFT REJECTIONCOMPLEMENT-
OXIDATIVE PHOSPHORYLATIONDNAREPAIR G2M CHECKPOINTE2F TARGETS OTIC SPINDLE-

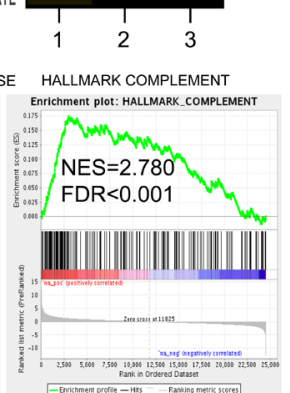

Figure 3. TSC1 deletion alters the expression of genes associated with inflammation in embryo renal PTCs and this is reversed by rapamycin treatment during pregnancy. (A) Kidneys from E18.5 control and TSC1-null mice with and without rapamycin, given as in Figure 2A, were removed, cells dissociated, and proximal tubule cells stained with PE-conjugated prominin-1 antibody. Prominin-1-positive cells were monitored and sorted by FACS to isolate PTCs. Representative analyses of 3 samples for each group. (B) Heatmap visualization showing relative gene expression in sorted PTCs from control and Six2 $\mathrm{Cre}^{\mathrm{tg} / \mathrm{TS}} \mathrm{TSC}^{\mathrm{fl} / \mathrm{fl}}$ embryos without (DMSO) and with rapamycin given during pregnancy. $n=3$ in each group. (C) Validation by qPCR of changes in expression of genes associated with inflammatory response and macrophage polarization that were chosen based on B and C. $n=4$ in each group. One-way ANOVA statistical analysis was used followed by Duncan's post hoc test. ${ }^{*} P<0.05$. (D) Heatmap of potential pathways affected by TSC1 deletion and the effect of rapamycin treatment on these pathways in PTCs. The changes in pathways were calculated according to the GSEA in B. The number 1 represents the ratio

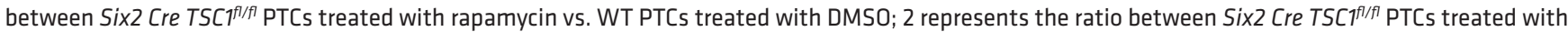
DMSO vs. WT PTCs treated with DMSO; and 3 represents the ratio between Six2 Cre TSC1f//fl PTCs treated with rapamycin vs. Six2 Cre TSC1 fl/fl PTCs treated with DMSO. (E) GSEA analysis of the inflammatory response and complement pathways in Six2 Cre TSC ff/fl PTCs compared with control WT PTCs. TSC, tuberous sclerosis complex; PTCs, proximal tubular cells; GSEA, gene set enrichment analysis; TSC, tuberous sclerosis complex.

reported in animal models $(39,41)$. Our findings suggest that the use of antiinflammatory agents such as corticosteroids may reduce kidney damage induced by cystogenesis. Indeed, corticosteroids such as dexamethasone and betamethasone are commonly used during pregnancy and may therefore serve as an alternative approach for the treatment of TSC.

Previous studies demonstrated that the catabolic effect of glucocorticoids on skeletal muscle is also mediated by inhibition of the mTOR pathway. Steroid administration led to decreased levels of phosphorylated 4EBP1 and S6 kinase 1, the main downstream targets of mTORC1. It has been suggested that glucocorticoids enhance the expression of REDD1, a mTORC1 suppressor (42-44). Our study is a report of the effect of glucocorticoids on TSC disease, such as cystic kidney disease. We showed that the effect of glucocorticoids is direct through modulation of $\mathrm{mTORC} 1$ activity as well as by the inhibition of the inflammatory response, which is essential for TSC cystogenesis (42-44).

Our results indicated increased c-Myc expression in TSC cystic epithelial cells that is mTOR dependent. A relationship between the hamartin-tuberin complex, acting as a tumor suppressor gene, and c-Myc has been studied in tumors (45-47). c-Myc, as a potent oncogene, induces expression of genes involved in cell proliferation in various tumors. On the one hand, c-Myc is a direct repressor of TSC2 expression-encoding tuberin, the coprotein of hamartin. On the other hand, the loss of TSC, by loss of tuberin, enhances the expression of c-Myc, thereby causing an oncogenic loop. The effect of loss of TSC is mediated by hyperactivation of mTORC1. mTORC1 is required for proper translation of c-Myc 
A
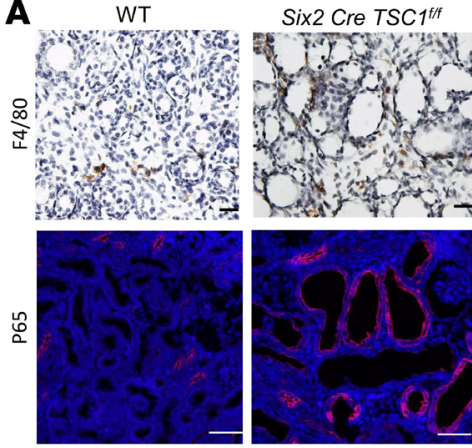

B
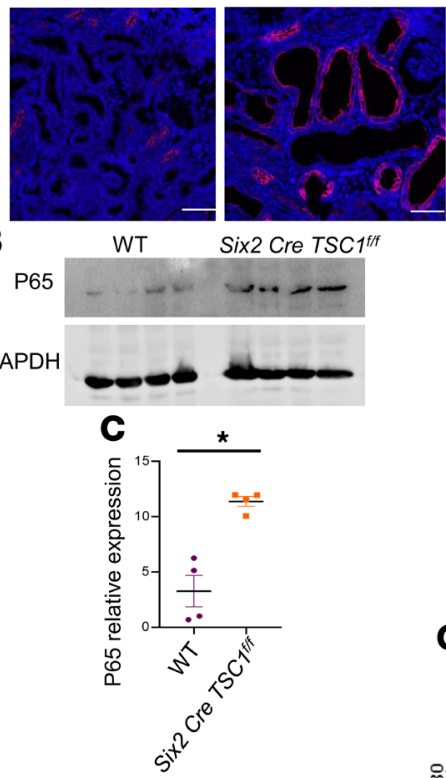

D
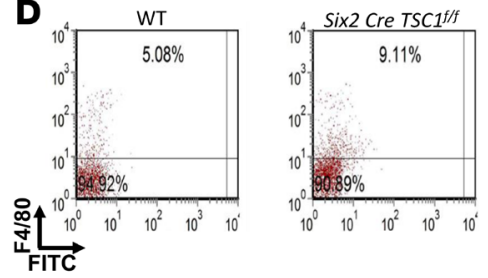

E
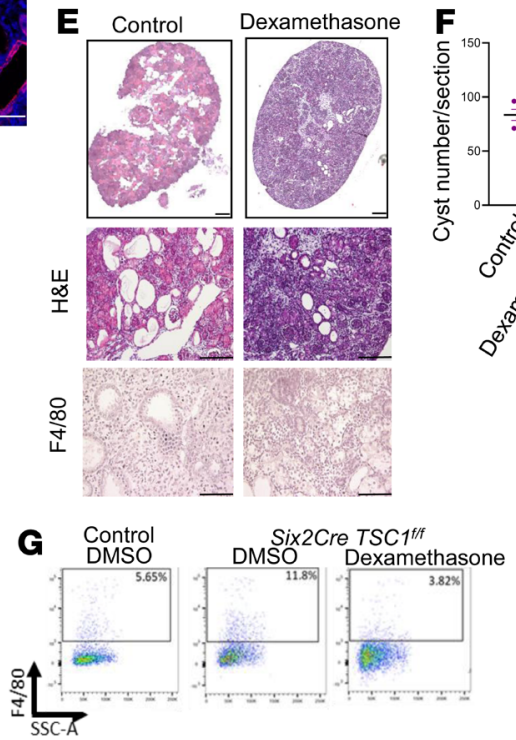
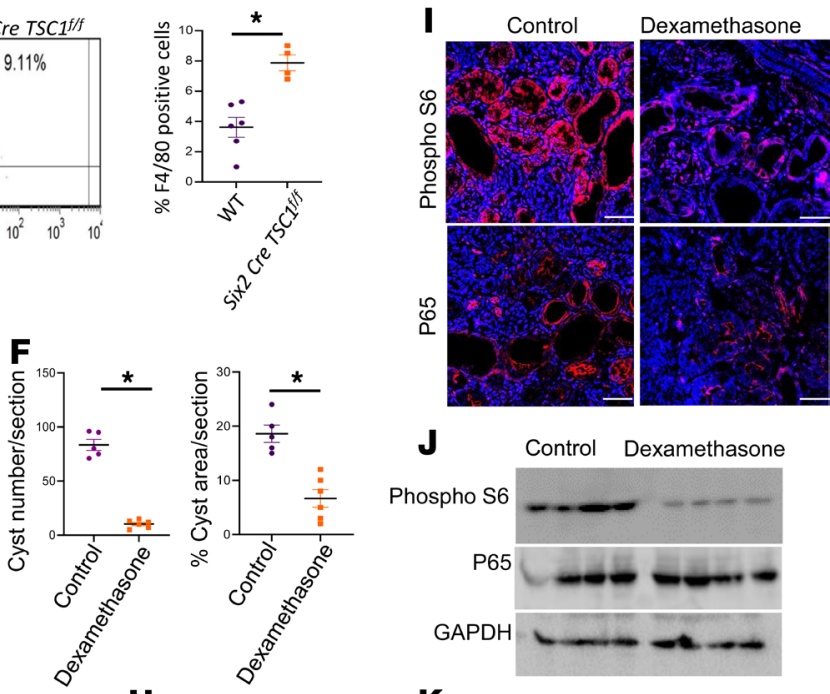

J

Control Dexamethasone

Phospho S6
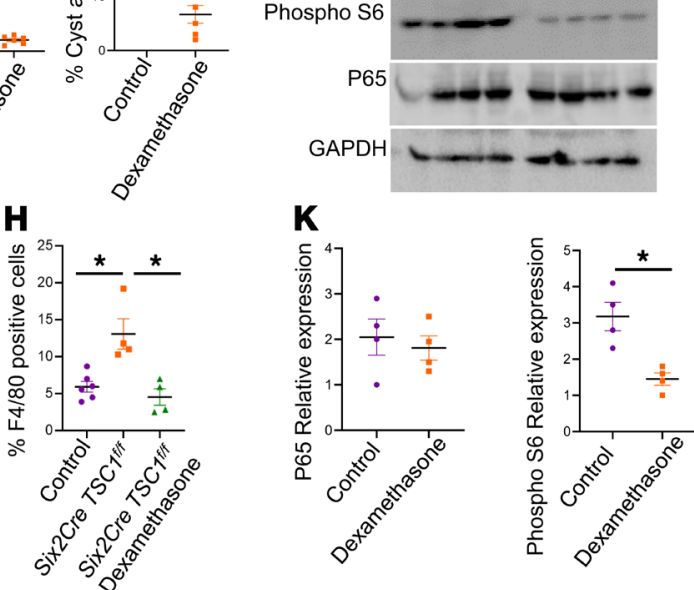

Figure 4. Dexamethasone administered during pregnancy reduces cyst formation and mTORC1 activity in TSC mice. (A) Renal sections of control and Six2 $\mathrm{Cre}^{\mathrm{tg} /+} \mathrm{TSC}^{\mathrm{fl/f}}$ mice (PO) were stained with anti-F4/80 marker (macrophages) and anti-NF-KB P65. Scale bar: $50 \mu \mathrm{m}$. $n=3$. (B) Western blot for P65 and control GAPDH in homogenized kidneys from control and TSC1 KO mice. (C) Quantification of the Western blot in B. ${ }^{*} P<0.05 . n=$ 4. (D) Kidneys from mice as in A were removed. Cells were dissociated and stained with APC-conjugated F4/80. The percentage of F4/80+ cells was determined by FACS. WT $(n=6)$, Six2 Cre TSC $f^{f / f l}(n=4) .{ }^{*} P<0.05$. (E) Representative H\&E staining of kidney sections from Six2 $C r e^{t g /+} T S C 7^{f / / f l}$ mice treated with dexamethasone and control (PBS), showing reduced cyst formation after dexamethasone. Scale bar $=500 \mu \mathrm{m}$. (F) Quantification of the cyst area and number per section in the different groups as in F. Dexamethasone $(n=6)$ and control $(n=5)$. ${ }^{*}<<0.05$. (C) Representative FACS analysis. Kidneys of WT, Six2 Cre TSC $f^{f / f l}$ treated with either PBS or dexamethasone as indicated were dissociated and stained with APC-conjugated F4/80 and subjected to FACS analysis. (H) Quantification of the percentage of F4/80+ cells in each group as in G. WT $(n=5)$, Six2 Cre TSC $7^{f / f / l}$ treated with PBS $(n=4)$, and Six2 Cre TSC ${ }^{f / f f}$ treated with dexamethasone $(n=4) .{ }^{*} P<0.05$. (I) Sections as in E were stained for pS6 and P65. Scale bar: $50 \mu \mathrm{m}$. $n=3$. (J) Kidneys were homogenized as in $\mathbf{E}$ and the expression of P65, pS6, and GAPDH were analyzed by Western blots $(n=4)$. (K) Quantification of the Western blots as shown in $\mathbf{J} .{ }^{*} P<0.05$. Unpaired $t$ test was used for $\mathbf{C}, \mathbf{D}, \mathbf{F}$, and $\mathbf{K}$, and 1 -way ANOVA statistical analysis was used followed by Duncan's post hoc test for $\mathbf{H}$. mTORC1, mTOR complex 1; TSC, tuberous sclerosis complex.

as well as its posttranscriptional modification to enhance c-Myc stability (48). Lately, a similar relationship of c-Myc with hamartin encoded by TSC1 was revealed in c-Myc-driven tumors such as Burkitt's lymphoma, also in a mTORC1 dependent manner (46). Indeed, renal cystogenesis is characterized by an increased proliferation rate of tubular cells. Analysis of ADPKD kidney biopsies also confirmed increased c-Myc expression specifically in the cystic epithelial cells concurrent with an increased proliferation rate $(29,30,49,50)$. Inhibition of c-Myc significantly reduced cystogenesis in vivo (51). Here, we report that interruption of the hamartin-tuberin complex in NPCs by TSC1 deletion during pregnancy is associated with high c-Myc activity and proliferation rate in TSC cyst-lining cells.

The role of inflammation in TSC manifestation was also demonstrated in TSC-related tumors. Rapamycin leads to only partial regression of TSC tumors, including AML, with a high risk of relapse of the tumor after cessation of rapamycin therapy. The adequate response of the immune system to the tumors may also be impaired by the effect of rapamycin on the immune system. Immunotherapy by PD-1 and CTLA-4 blockade leads to substantial regression of TSC-related tumors with long-term remission, which may be further improved by combination therapy with mTORC1 inhibitors (52-57).

Our findings on the role of inflammation in TSC cystic disease are compatible with new evidence demonstrating that macrophages play a central role in cyst formation and progression in $\operatorname{ADPKD}(58,59)$. 
A

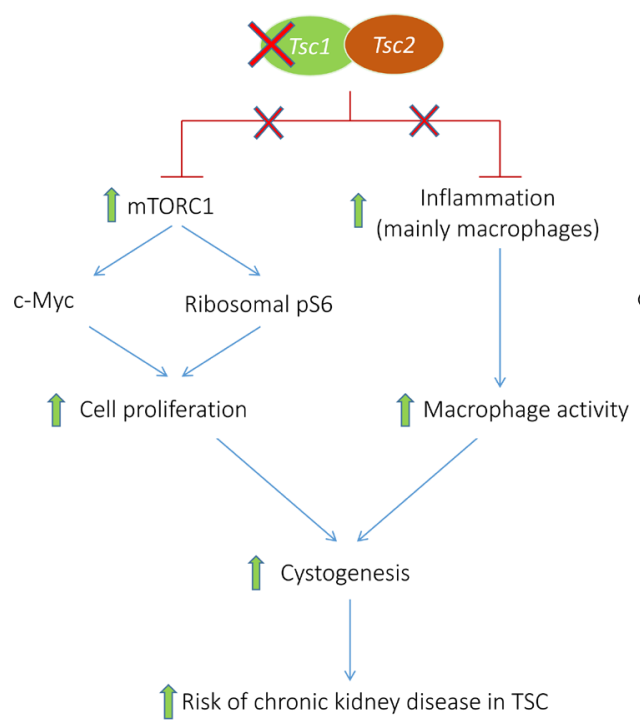

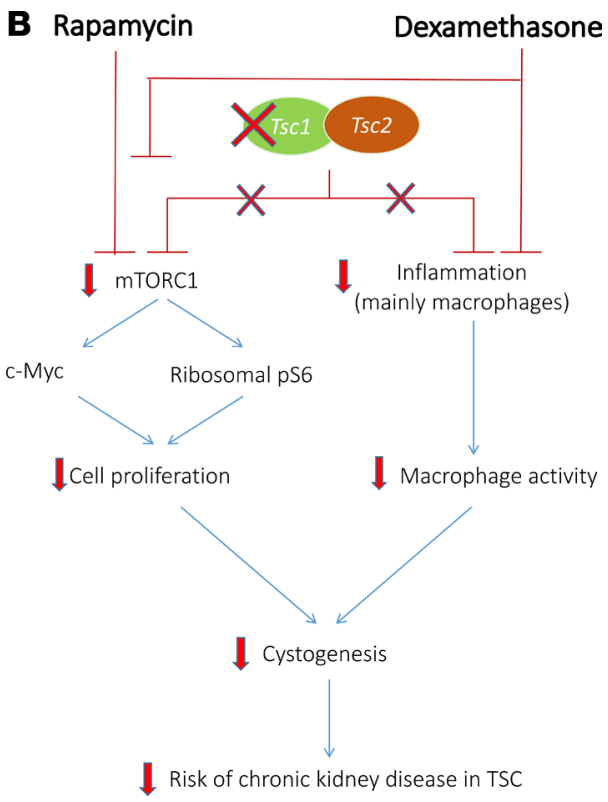

Figure 5. Scheme summarizing the proposed mechanism by which rapamycin and dexamethasone administration during pregnancy prevent cystic kidney disease in TSC. (A) TSC1 deletion in nephron progenitor cells leads to increased mTORC 1 activity and an inflammatory response in the nephron. Cysts arise from proximal tubular cells in the developing nephrons as a result of a high rate of cell proliferation and increased macrophage activity, which increase the risk of chronic kidney disease in adult patients with TSC. (B) By reducing mTORC1 activity, rapamycin leads to decreased ribosomal translation and c-Myc expression, thereby decreasing the proliferation rate of the tubular cells. Dexamethasone ameliorates cyst formation by inhibiting both mTORC1 and the inflammatory response in the cysts. TSC, tuberous sclerosis complex; mTORC1, mTOR complex 1 .

Previous studies have shown that infiltrating macrophages are initially of the proinflammatory classically activated M1-like type, which exacerbate tubular injury due to their release of reactive oxygen and nitrogen intermediates (60). Later, the M1-like macrophages are converted to alternatively activated M2-like macrophages, which then stimulate the proliferation of tubular epithelial cells for the apparent purpose of facilitating the repair of the sustained injury. Whether the same molecular mechanisms by which macrophages induce cytogenesis in ADPKD are activated in TSC awaits further investigation.

We demonstrated that hyperactivation of mTOR plays a central role in TSC cystic kidney disease by increasing PTC proliferation rate, which is also mediated by enhancing c-Myc activity. We also showed that increased inflammation, mainly macrophagic infiltration, contributes to cyst formation in TSC, as demonstrated in other cystic kidney disease. Steroid administration diminishes cystogenesis by not only modulating the inflammatory response but also interfering with mTORC1 activity, as has been demonstrated in other organs. Our findings raise an alternative strategy for early intervention during pregnancy to prevent long-term kidney disease as a result of cyst formation in TSC. Our data may also contribute to the understanding of other aspects of TSC disease, such as AML, in the kidney as well as in other organs.

\section{Methods}

Animals. All mice were maintained in the Hebrew University Specific-Pathogen-Free (SPF) Animal Facility Unit. The transgenic mice were a gift from Raphael Kopan's lab from the division of developmental biology from Cincinnati Children's Hospital Medical Center. The following CD1 transgenic mice lines were used: $\mathrm{Tg}$ (Six2-EGFP/cre) $1 \mathrm{Amc}$ (herein Six2 Cre ${ }^{\mathrm{tg} /+}$ ) and TSC1 $1^{\mathrm{fl} / \mathrm{ll}}$ (8). For heterozygous deletion of TSC1 in NPCs, 6to 8-week-old Six2 $\mathrm{Cre}^{\text {tg/+ }}$ male mice were mated with 6- to 8-week-old TSC1/flf females. To generate homozygous TSC1 deletion (Six2 $\mathrm{Cr}^{\mathrm{tg} /+} \mathrm{TSC1}^{\mathrm{fl} / \mathrm{f}}$ ) mice, 6- to 8-week-old heterozygous Six2 $\mathrm{Cre}^{\mathrm{tg} /+} \mathrm{TSC1}^{\mathrm{fl} /+}$ males were backcrossed with 6- to 8-week-old $T S C 1^{f / f l}$ females. The pregnancy date was determined by vaginal plug expulsion. The morning of plug detection was designated as day 0.5 of pregnancy. At different embryonic dates, pregnant females were sacrificed using $\mathrm{CO}_{2}$ and cervical dislocation. The embryos and newborn pups were dissected and kidneys were excised for histopathology evaluation. In some experiments, rapamycin $(0.2 \mathrm{mg} / \mathrm{kg})$, dexamethasone $(0.1 \mathrm{mg} / \mathrm{kg})$, or vehicle (DMSO or PBS, respectively) were i.p. injected.

Histology. Embryonic or newborn mouse kidneys were dissected on ice-cold PBS and fixed overnight in fresh $4 \%$ formaldehyde in PBS. Kidneys were embedded in paraffin for sectioning. For overall histology, tissue sections were stained with hematoxylin/eosin and PAS staining. IHC and IF staining were performed as previously described $(8,61)$. Briefly, paraffin-embedded tissue sections $(4-6 \mu \mathrm{m})$ were deparaffinized, hydrated, and incubated overnight at $4^{\circ} \mathrm{C}$ with the following antibodies according to the manufacturer's instructions: biotin anti-LTL (B-1325, Vector); rabbit anti-phospho-S6 ribosomal protein (2211, Cell Signaling Technology); mouse anti-c-Myc (Sc-40, Santa Cruz Biotechnology); 
rabbit anti-Ki-67 (MU297-UC, Biogenex); rabbit anti-P65 (8242, Cell Signaling Technology); and rat anti-F4/80 (MCA497, Bio-Rad). For IHC, DAB reagent was applied after incubation with the appropriate HRP-bound secondary antibody. For IF staining, the sections were incubated with either Cy3-conjugated goat anti-rabbit or Cy5-conjugated goat anti-mouse antibody according to the manufacturer's instructions (Jackson ImmunoResearch). The sections were visualized with a confocal A1R microscope (original magnification, $\times 20$ ) unless stated otherwise.

Cystic index. The cyst number for a given section area and the cyst area ratio compared with total kidney section area were evaluated with NIH ImageJ analysis software.

Western blotting. Kidneys were extracted and homogenized in cold RIPA buffer containing $150 \mathrm{Mm}$ $\mathrm{NaCl}, 1 \% \mathrm{NP} 40,0.5 \%$ sodium deoxycholate, $0.1 \% \mathrm{SDS}$, and $25 \mathrm{Mm}$ Tris (pH 7.4) and supplemented with protease/phosphatase inhibitors (4906837001, Merck). An equal amount of protein extract was analyzed by SDS-PAGE, as previously described (61), using the following antibodies as described above, according to the manufacturer's instructions: rabbit anti-phospho-S6 ribosomal protein; mouse anti-c-Myc (Sc-40, Santa Cruz Biotechnology); rabbit anti-P65 (8242, Cell Signaling Technology); and mouse anti-GAPDH (MAB374, MilliporeSigma). See complete unedited blots in the supplemental material.

FACS of PTCS. Kidneys were excised in ice-cold HBSS buffer. The kidneys were sliced and chopped into pieces $(\sim 0.5-1 \mathrm{~mm})$ on ice using a surgical scalpel. The chopped kidneys were transferred into HBSS solution containing $1 \mu \mathrm{g} / \mu \mathrm{L}$ collagenase/dispase (10269638001, MilliporeSigma) and incubated for 25 minutes at $37^{\circ} \mathrm{C}$. The cells were filtered through a $40-\mu \mathrm{m}$ nylon cell strainer (Corning) and washed twice with cold HBSS. For PTC isolation, the cells were stained with PE-conjugated antiCD133/prominin-1 antibody (12-1331-82, Invitrogen) according to manufacturer's instructions. $\mathrm{PE}^{+}$ cells were isolated by cell flow cytometry-based cell sorting (Hebrew University Faculty of Medicine Core Facility). The cells were washed and total RNA was extracted using peqGOLD TriFast (PeqLab Biotechnologies). For FACS analysis of $\mathrm{F} 4 / 80^{+}$cells, kidneys were chopped as described above and stained with APC-conjugated anti-F4/80 antibody (130-117-509, Macs Miltenyi Biotec). The cells were washed twice with HBSS before analysis by LSRII flow cytometry.

RNA sequencing and pathway analysis. The RNA quality was evaluated in TapeStation using the RNA ScreenTape kit (Agilent Technologies) and quantified using a Qubit apparatus (Qubit DNA HS Assay kit, Invitrogen). Libraries were prepared from RNA samples using a KAPA Stranded mRNASeq Kit (KK8401, Roche). The libraries were barcoded and pooled for multiplex sequencing (1.5 pM total, including $1.5 \%$ PhiX control library). The pooled cDNA was loaded on a NextSeq 500 High Output v2 kit (75 cycles) cartridge (Illumina) and sequenced on Illumina NextSeq 500 System, using sequencing conditions of 75 cycles, single-read. Library preparation and sequencing were performed at the Core Facility of the Hebrew University Faculty of Medicine. For further validation, RNA was extracted and used for quantitative PCR (qPCR) with the following primers: IL-6 forward, 5'-CTCTGCAAGAGACTTCCATCCA-3'; IL-6 reverse, 5'-GACAGGTCTGTTGGGAGTGG-3'; TNFA forward, 5'-TAGCCCACGTCGTAGCAAAC-3'; TNFA reverse, 5'-ACAAGGTACAACCCATCGGC-3'; CXCL10 forward, 5'-ATGACGGGCCAGTGAGAATG-3'; CXCL10 reverse, 5'-TCGTGGCAATGATCTCAACAC-3'; and CCL4 forward, 5'-CTGTGCAAACCTAACCCCGA-3'; CCL4 reverse, 5'-AGGGTCAGAGCCCATTGGT-3'.

Gene set enrichment analysis. Differential expression data from TSC1-KO versus TSC1-KO-Rapamycin were subjected to gene set enrichment analysis (GSEA, Broad Institute). GSEA uses ranked differential expression data (cutoff independent) to determine whether a priori-defined sets of genes show statistically significant and concordant differences between 2 biological states. GSEA was run against the hallmark gene sets collection from the molecular signatures database.

Statistics. The numbers of biological samples were determined based on effect size or sample variation. No statistical method was used to predetermine the sample size. No animals or samples were excluded from any analysis. Animals were randomly assigned to groups for in vivo studies; no formal randomization method was applied when assigning animals for treatment. Values are reported as means \pm SEM unless otherwise stated. The data were analyzed by a Student's 2-tailed $t$ test. In experiments as indicated, 1-way ANOVA analysis was performed followed by Duncan's post hoc test. The significance was set at a $P$ value of less than 0.05. The data are presented using the GraphPad Prism version 7.

Study approval. All animal studies were carried out in compliance with the ethical regulations approved by the Animal Care Committee of the Hebrew University Medical School (ethical approval no. MD-17-15368-4). 


\section{Author contributions}

$\mathrm{MN}$ and $\mathrm{OV}$ conceived the study, designed the experiment, and wrote the manuscript. MN conducted most of the experiments and supervised the rest of the experiments. YM and ER conducted some of the experiments. KM helped to conceive the study and critically reviewed the manuscript.

\section{Acknowledgments}

This work was supported by grants from the Israel Science Foundation (to OV, 2358/18) and institutional grants (startup and bridging) from the Hadassah-Hebrew University Medical Center (to OV). OV and MN are research associates of the Wohl's Translation Research Institute at Hadassah-Hebrew University Medical Center. The graphical abstract was created using Biorender.com.

Address correspondence to: Oded Volovelsky, Pediatric Nephrology Unit, Hadassah-Hebrew University Medical Center, Ein-Kerem Campus, Jerusalem, 91120, POB 12000, Israel. Phone: 972.2.6777023; Email: odedvo@hadassah.org.il.

1. Osborne JP, Fryer A, Webb D. Epidemiology of tuberous sclerosis. Ann N Y Acad Sci. 1991;615:125-127.

2. Crino PB, Nathanson KL, Henske EP. The tuberous sclerosis complex. N Engl J Med. 2006;355(13):1345-1356.

3. Yates JR. Tuberous sclerosis. Eur J Hum Genet. 2006;14(10):1065-1073.

4. Hong CH, Tu HP, Lin JR, Lee CH. An estimation of the incidence of tuberous sclerosis complex in a nationwide retrospective cohort study (1997-2010). Br J Dermatol. 2016;174(6):1282-1289.

5. European Chromosome 16 Tuberous Sclerosis Consortium. Identification and characterization of the tuberous sclerosis gene on chromosome 16. Cell. 1993;75(7):1305-1315.

6. van Slegtenhorst M, et al. Identification of the tuberous sclerosis gene TSC1 on chromosome 9q34. Science. 1997;277(5327):805-808.

7. Dabora SL, et al. Mutational analysis in a cohort of 224 tuberous sclerosis patients indicates increased severity of TSC2, compared with TSC1, disease in multiple organs. Am J Hum Genet. 2001;68(1):64-80.

8. Volovelsky O, et al. Hamartin regulates cessation of mouse nephrogenesis independently of Mtor. Proc Natl Acad Sci USA. 2018;115(23):5998-6003.

9. Ingelfinger JR. Tackling Tsc1 to promote nephrogenesis. N Engl J Med. 2018;379(25):2476-2478.

10. Hartman TR, et al. The tuberous sclerosis proteins regulate formation of the primary cilium via a rapamycin-insensitive and polycystin 1-independent pathway. Hum Mol Genet. 2009;18(1):151-163.

11. Karbowniczek M, et al. The evolutionarily conserved TSC/Rheb pathway activates Notch in tuberous sclerosis complex and Drosophila external sensory organ development. J Clin Invest. 2010;120(1):93-102.

12. Li C, et al. Tuberin Regulates Prostaglandin Receptor-Mediated Viability, via Rheb, in mTORC1-Hyperactive Cells. Mol Cancer Res. 2017;15(10):1318-1330.

13. Alves MM, et al. PAK2 is an effector of TSC1/2 signaling independent of mTOR and a potential therapeutic target for Tuberous Sclerosis Complex. Sci Rep. 2015;5:14534.

14. Thien A, et al. TSC1 activates TGF- $\beta$-Smad2/3 signaling in growth arrest and epithelial-to-mesenchymal transition. Dev Cell. 2015;32(5):617-630.

15. Brugarolas JB, Vazquez F, Reddy A, Sellers WR, Kaelin WG. TSC2 regulates VEGF through mTOR-dependent and -independent pathways. Cancer Cell. 2003;4(2):147-158.

16. Bissler JJ, et al. Sirolimus for angiomyolipoma in tuberous sclerosis complex or lymphangioleiomyomatosis. $N$ Engl J Med. 2008;358(2):140-151.

17. Yalon M, Ben-Sira L, Constantini S, Toren A. Regression of subependymal giant cell astrocytomas with RAD001 (Everolimus) in tuberous sclerosis complex. Childs Nerv Syst. 2011;27(1):179-181.

18. Kingswood C, et al. The clinical profile of tuberous sclerosis complex (TSC) in the United Kingdom: A retrospective cohort study in the Clinical Practice Research Datalink (CPRD). Eur J Paediatr Neurol. 2016;20(2):296-308.

19. Kingswood JC, et al. TuberOus SClerosis registry to increase disease Awareness (TOSCA) - baseline data on 2093 patients. Orphanet J Rare Dis. 2017;12(1):2.

20. Shepherd CW, Gomez MR, Lie JT, Crowson CS. Causes of death in patients with tuberous sclerosis. Mayo Clin Proc. 1991;66(8):792-796.

21. Dixon BP, Hulbert JC, Bissler JJ. Tuberous sclerosis complex renal disease. Nephron Exp Nephrol. 2011;118(1):e15-e20.

22. Siroky BJ, Yin H, Bissler JJ. Clinical and molecular insights into tuberous sclerosis complex renal disease. Pediatr Nephrol. 2011;26(6):839-852.

23. Kingswood JC, et al. Review of the Tuberous Sclerosis Renal Guidelines from the 2012 Consensus Conference: Current Data and Future Study. Nephron. 2016;134(2):51-58.

24. Sampson JR, et al. Renal cystic disease in tuberous sclerosis: role of the polycystic kidney disease 1 gene. Am J Hum Genet. 1997;61(4):843-851.

25. Consugar MB, et al. Characterization of large rearrangements in autosomal dominant polycystic kidney disease and the PKD1/ TSC2 contiguous gene syndrome. Kidney Int. 2008;74(11):1468-1479.

26. Bissler JJ, et al. Everolimus for angiomyolipoma associated with tuberous sclerosis complex or sporadic lymphangioleiomyomatosis (EXIST-2): a multicentre, randomised, double-blind, placebo-controlled trial. Lancet. 2013;381(9869):817-824.

27. Lam HC, Siroky BJ, Henske EP. Renal disease in tuberous sclerosis complex: pathogenesis and therapy. Nat Rev Nephrol. 
2018;14(11):704-716.

28. Wu H, Chen J, Xu J, Dong Z, Meyuhas O, Chen JK. Blocking rpS6 Phosphorylation Exacerbates Tsc1 Deletion-Induced Kidney Growth. J Am Soc Nephrol. 2016;27(4):1145-1158

29. Trudel M, et al. C-myc-induced apoptosis in polycystic kidney disease is Bcl-2 and p53 independent. J Exp Med. 1997;186(11):1873-1884.

30. Parrot C, Kurbegovic A, Yao G, Couillard M, Côté O, Trudel M. c-Myc is a regulator of the PKD1 gene and PC1-induced pathogenesis. Hum Mol Genet. 2019;28(5):751-763.

31. Legouis D, et al. Ex vivo analysis of renal proximal tubular cells. BMC Cell Biol. 2015;16:12.

32. Fernando MR, Reyes JL, Iannuzzi J, Leung G, McKay DM. The pro-inflammatory cytokine, interleukin- 6 , enhances the polarization of alternatively activated macrophages. PLoS One. 2014;9(4):e94188.

33. Wu X, et al. TNF-a mediated inflammatory macrophage polarization contributes to the pathogenesis of steroid-induced osteonecrosis in mice. Int J Immunopathol Pharmacol. 2015;28(3):351-361.

34. Petrovic-Djergovic D, Popovic M, Chittiprol S, Cortado H, Ransom RF, Partida-Sánchez S. CXCL10 induces the recruitment of monocyte-derived macrophages into kidney, which aggravate puromycin aminonucleoside nephrosis. Clin Exp Immunol. 2015;180(2):305-315

35. Crowther CA, et al. Maternal intramuscular dexamethasone versus betamethasone before preterm birth (ASTEROID): a multicentre, double-blind, randomised controlled trial. Lancet Child Adolesc Health. 2019;3(11):769-780.

36. Au KS, Hebert AA, Roach ES, Northrup H. Complete inactivation of the TSC2 gene leads to formation of hamartomas. Am J Hum Genet. 1999;65(6):1790-1795.

37. Lam HC, Nijmeh J, Henske EP. New developments in the genetics and pathogenesis of tumours in tuberous sclerosis complex. J Pathol. 2017;241(2):219-225.

38. Hung TH, Hsieh TT, Wu CP, Li MJ, Yeh YL, Chen SF. Mammalian target of rapamycin signaling is a mechanistic link between increased endoplasmic reticulum stress and autophagy in the placentas of pregnancies complicated by growth restriction. Placenta. 2017;60:9-20.

39. Park H, Chang CS, Choi SJ, Oh SY, Roh CR. Sirolimus therapy for fetal cardiac rhabdomyoma in a pregnant woman with tuberous sclerosis. Obstet Gynecol Sci. 2019;62(4):280-284

40. Anderl S, Freeland M, Kwiatkowski DJ, Goto J. Therapeutic value of prenatal rapamycin treatment in a mouse brain model of tuberous sclerosis complex. Hum Mol Genet. 2011;20(23):4597-4604.

41. Colla L, et al. Immunosuppression in pregnant women with renal disease: review of the latest evidence in the biologics era. J Nephrol. 2018;31(3):361-383.

42. Shah OJ, Anthony JC, Kimball SR, Jefferson LS. Glucocorticoids oppose translational control by leucine in skeletal muscle. Am J Physiol Endocrinol Metab. 2000;279(5):E1185-E1190.

43. Shah OJ, Kimball SR, Jefferson LS. Acute attenuation of translation initiation and protein synthesis by glucocorticoids in skeletal muscle. Am J Physiol Endocrinol Metab. 2000;278(1):E76-E82.

44. Wang H, Kubica N, Ellisen LW, Jefferson LS, Kimball SR. Dexamethasone represses signaling through the mammalian target of rapamycin in muscle cells by enhancing expression of REDD1. J Biol Chem. 2006;281(51):39128-39134.

45. Schmidt EV, Ravitz MJ, Chen L, Lynch M. Growth controls connect: interactions between c-myc and the tuberous sclerosis complex-mTOR pathway. Cell Cycle. 2009;8(9):1344-1351.

46. Hartleben G, et al. Tuberous sclerosis complex is required for tumor maintenance in MYC-driven Burkitt's lymphoma. EMBO J. 2018;37(21):e98589.

47. Babcock JT, Nguyen HB, He Y, Hendricks JW, Wek RC, Quilliam LA. Mammalian target of rapamycin complex 1 (mTORC1) enhances bortezomib-induced death in tuberous sclerosis complex (TSC)-null cells by a c-MYC-dependent induction of the unfolded protein response. J Biol Chem. 2013;288(22):15687-15698.

48. Wu L, et al. Loss of FOXP3 and TSC1 Accelerates Prostate Cancer Progression through Synergistic Transcriptional and Posttranslational Regulation of c-MYC. Cancer Res. 2019;79(7):1413-1425.

49. Husson $\mathrm{H}$, et al. New insights into ADPKD molecular pathways using combination of SAGE and microarray technologies. Genomics. 2004;84(3):497-510

50. Song X, et al. Systems biology of autosomal dominant polycystic kidney disease (ADPKD): computational identification of gene expression pathways and integrated regulatory networks. Hum Mol Genet. 2009;18(13):2328-2343.

51. Zhou X, Fan LX, Peters DJ, Trudel M, Bradner JE, Li X. Therapeutic targeting of BET bromodomain protein, Brd4, delays cyst growth in ADPKD. Hum Mol Genet. 2015;24(14):3982-3993.

52. Onda H, Lueck A, Marks PW, Warren HB, Kwiatkowski DJ. Tsc2(+/-) mice develop tumors in multiple sites that express gelsolin and are influenced by genetic background. J Clin Invest. 1999;104(6):687-695.

53. Li Q, et al. Regulating mammalian target of rapamycin to tune vaccination-induced CD8(+) T cell responses for tumor immunity. J Immunol. 2012;188(7):3080-3087.

54. Berezhnoy A, Castro I, Levay A, Malek TR, Gilboa E. Aptamer-targeted inhibition of mTOR in T cells enhances antitumor immunity. J Clin Invest. 2014;124(1):188-197.

55. Chaoul N, et al. Rapamycin Impairs Antitumor CD8+ T-cell Responses and Vaccine-Induced Tumor Eradication. Cancer Res. 2015;75(16):3279-3291.

56. Liu HJ, et al. TSC2-deficient tumors have evidence of T cell exhaustion and respond to anti-PD-1/anti-CTLA-4 immunotherapy. JCI Insight. 2018;3(8):98674.

57. Liu HJ, Krymskaya VP, Henske EP. Immunotherapy for Lymphangioleiomyomatosis and Tuberous Sclerosis: Progress and Future Directions. Chest. 2019;156(6):1062-1067.

58. Swenson-Fields KI, et al. Macrophages promote polycystic kidney disease progression. Kidney Int. 2013;83(5):855-864.

59. Cassini MF, et al. Mcp1 Promotes Macrophage-Dependent Cyst Expansion in Autosomal Dominant Polycystic Kidney Disease. J Am Soc Nephrol. 2018;29(10):2471-2481

60. Lee S, et al. Distinct macrophage phenotypes contribute to kidney injury and repair. J Am Soc Nephrol. 2011;22(2):317-326.

61. Volovelsky O, et al. Phosphorylation of Ribosomal Protein S6 Mediates Mammalian Target of Rapamycin Complex 1-Induced Parathyroid Cell Proliferation in Secondary Hyperparathyroidism. J Am Soc Nephrol. 2016;27(4):1091-1101. 